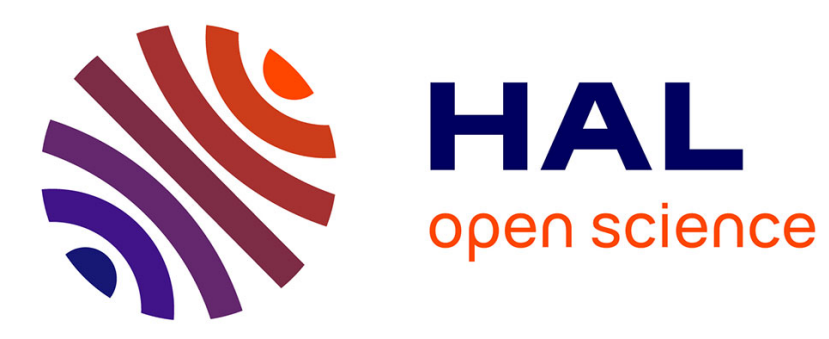

\title{
Towards unlocking the full potential of Multileaf Collimators
}

\author{
Guillaume Blin, Paul Morel, Romeo Rizzi, Stéphane Vialette
}

\section{To cite this version:}

Guillaume Blin, Paul Morel, Romeo Rizzi, Stéphane Vialette. Towards unlocking the full potential of Multileaf Collimators. 40th International Conference on Current Trends in Theory and Practice of Computer Science (SOFSEM), Jan 2014, High Tatras, Slovakia. pp.138-149. hal-00859708

\section{HAL Id: hal-00859708 https://hal.science/hal-00859708}

Submitted on 9 Sep 2013

HAL is a multi-disciplinary open access archive for the deposit and dissemination of scientific research documents, whether they are published or not. The documents may come from teaching and research institutions in France or abroad, or from public or private research centers.
L'archive ouverte pluridisciplinaire HAL, est destinée au dépôt et à la diffusion de documents scientifiques de niveau recherche, publiés ou non, émanant des établissements d'enseignement et de recherche français ou étrangers, des laboratoires publics ou privés. 


\title{
Towards unlocking the full potential of Multileaf Collimators ${ }^{\star}$
}

\author{
Guillaume Blin ${ }^{1}$, Paul Morel ${ }^{1}$ Romeo Rizzi ${ }^{2}$, and Stéphane Vialette ${ }^{1}$ \\ 1 Université Paris-Est, LIGM - UMR CNRS 8049, France. \\ \{gblin, paul.morel, vialette\}@univ-mlv.fr \\ 2 Department of Computer Science - University of Verona, Italy. \\ romeo.rizzi@univr.it
}

\begin{abstract}
A central problem in the delivery of intensity-modulated radiation therapy (IMRT) using a multileaf collimator (MLC) relies on finding a series of leaves configurations that can be shaped with the MLC to properly deliver a given treatment. In this paper, we analyse, from an algorithmic point of view, the power of using dual-layer MLCs and Rotating Collimators for this purpose.
\end{abstract}

\section{Radiation therapy planning}

Radiation therapy is one of the most commonly used cancer treatments and has been shown to be effective. The radiation treatment poses a tuning problem: the radiation needs to be effective enough to kill the tumor while sparing healthy tissues and organs close to the tumor - so-called organs at risk. Towards this goal, the design of radiation treatment has to be specifically customized for each patient. Once both tumor and organs at risks have been delineated, the radiation oncologist will prescribe minimal, maximal and mean irradiation quantity for each of them. The amount of radiation is measured in gray (Gy). For example, typical dose for a tumor ranges from 60 Gy to 80 Gy (a minimal dose that the treatment should achieve), whereas healthy organs should not receive more than a given threshold of radiation - for example, 20 Gy for lungs, 50 Gy for bones or $12 \mathrm{~Gy}$ for lens. Usually, the overall treatment dose is fractioned - e.g. 1.8 to 2 Gy per day, five days a week for an adult.

Each fraction is delivered by a linear accelerator (linac) using a cone beam that rotates around the patient; achieving a concentric irradiation converging in the tumor site. In the so-called "Step-And-Shoot" technique, the treatment design specifies some specific angles where the linac successively stops to irradiate the patient. For each of these angles, a specific intensity distribution accross the radiation beam (later on referred to as intensity matrix) is computed (for instance, with the multicriteria approach to radiation therapy planning of Hamacher and Küfer [6]) in order to achieve the desired overall dosage of the fraction. An illustration is provided in Figure 1a. The radiation generated by the accelerator is uniform. Therefore, in order to achieve the varying intensity,

\footnotetext{
* Work partially supported by ANR project BIRDS JCJC SIMI 2-2010
} 
this radiation needs to be modulated. For this purpose, each intensity matrix is delivered through a multileaf collimator (MLC). An MLC is a device composed of parallel pairs (referred to as rows) of facing tungsten strips (referred to as leaves) that can block the radiation by moving toward each other from left and right (see Figure 1c). However, radiation can pass through the open gap between the leaves endpoint. Each intensity matrix is realized by a sequence of MLC configurations (i.e. specific leaves positions for each row of the MLC) each of which is maintained for a certain amount of time (corresponding to the intensity). In the static case, the radiation is switched off while the collimator leaves are moving. The so-called gantry denotes the whole device including the linac and the MLC.

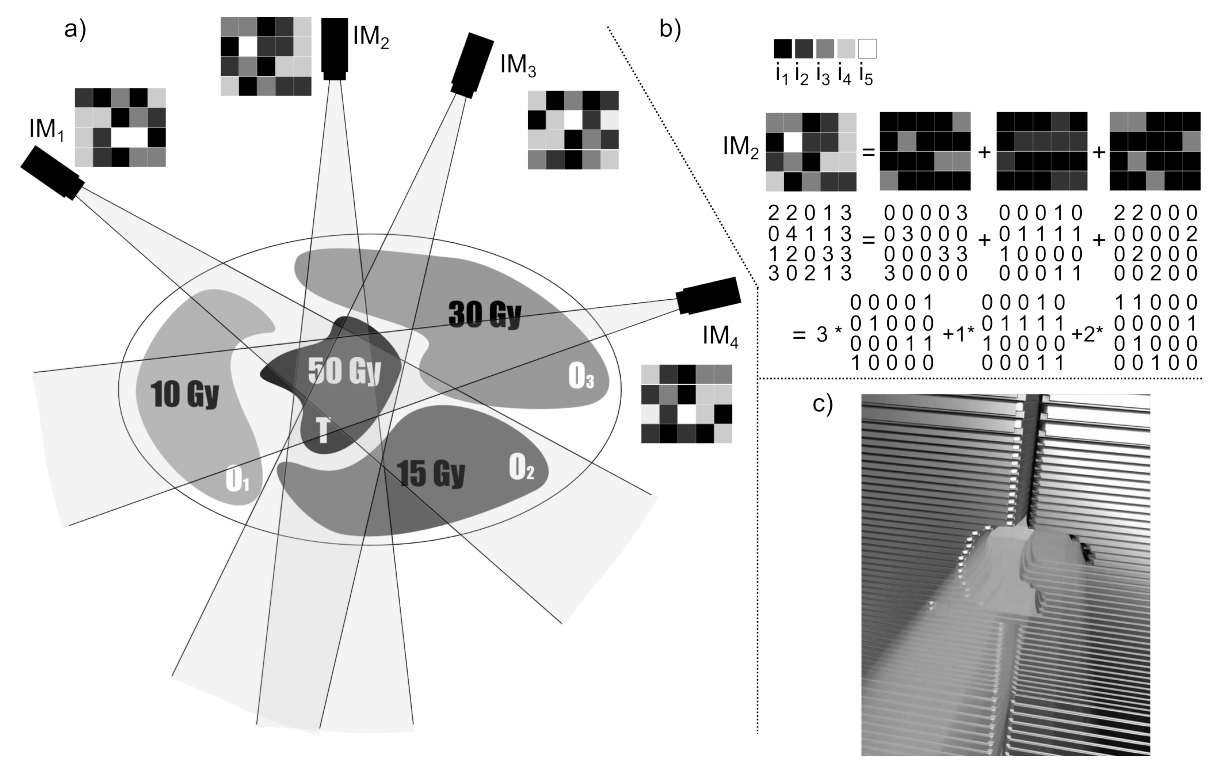

Fig. 1. a) IMRT with some intensity matrices - shown in grayscale coded grids with 5 intensities (the lighter the color the higher the radiation intensity). b) A realization of $I M_{2}$ with $i_{1}=0, i_{2}=1, i_{3}=2, i_{4}=3, i_{5}=4$. c) $M L C$ illustration from Varian

From an algorithmic point of view, the corresponding problem is a matrix decomposition problem where each intensity matrix is given as an integer matrix that has to be decomposed into a weighted sum of binary matrices (each binary matrix denotes an MLC configuration and the weight represents the associated intensity). These binary matrices are consecutive ones matrices (the 1 s occur consecutively as a single block in each row) since MLC leaves are moving from left and right sides of the device on each row. For example, the intensity matrix $I M_{2}$ of Figure $1 \mathrm{~b}$ can be decomposed into three configurations. Of course, there are many ways of decomposing a given intensity matrix. It is desirable to select 
the decomposition that can be delivered the most efficiently. The two main efficiency criteria that play a role are the total beam-on time, i.e., the total amount of time that the patient is being irradiated, and the total setup time, i.e., the total amount of time that is spent shaping the apertures. The former metric is proportional to the sum of intensities used in the decomposition, while the latter is (approximately) proportional to the number of matrices used in the decomposition. Although closely related, these two efficiency criteria are not equivalent. The intensity matrix $I M_{2}$ decomposition shows a decomposition using only 3 apertures with a beam-on time of 6 . However, the minimum beamon time for this intensity matrix is 5 , which can be realized by 5 apertures. Actually, it turns out that, while minimizing the total beam-on time is solvable in linear time, minimizing the total setup time is NP-hard for matrices with at least two rows [4]. This later result was strengthen by Baatar et. al [1] who proved that it is even strongly NP-hard, even for matrices with a single row.

Technology is running very fast and different MLC settings have been indeed proposed during the last decade. In this paper, we focus on algorithmic aspects of two technological variants: Intensity Modulated Radiation using Rotating Collimator and Multi-Layer Multileaf Collimator.

Rotating Collimator. Remember that the gantry is rotating around the patient to deliver the radiation. We consider here MLC rotations together with gantry rotations. Indeed, whereas most studies consider the problem of finding the most efficient collimator angle for each linac angle [2, 15, 13], some recent contributions tackle the use of collimator rotation in the decomposition of a given intensity matrix (i.e. for a given fixed linac angle) $[14,16,17,20,5,10,11,3]$. The practical efficiency of this latter technique was stated in [21]. From a Consecutive 1 Property $(\mathrm{C} 1 \mathrm{P})$ point of view, rotating collimator allows each intensity matrix to be decomposed in both row $\mathrm{C} 1 \mathrm{P}$ or column $\mathrm{C} 1 \mathrm{P}$ configurations. For example, decomposing the following intensity matrix with only row $\mathrm{C} 1 \mathrm{P}$ configurations requires at least 8 of them whereas only 6 configurations are needed if rotation is allowed (the last two configurations are column $\mathrm{C} 1 \mathrm{P}$ ).

$\left[\begin{array}{llll}1 & 4 & 2 & 5 \\ 1 & 3 & 3 & 2 \\ 1 & 3 & 5 & 5 \\ 6 & 4 & 6 & 0\end{array}\right]=\left[\begin{array}{llll}0 & 0 & 0 & 1 \\ 0 & 0 & 1 & 1 \\ 0 & 0 & 1 & 1 \\ 1 & 1 & 1 & 0\end{array}\right]+\left[\begin{array}{llll}0 & 0 & 0 & 1 \\ 0 & 1 & 1 & 1 \\ 0 & 0 & 1 & 1 \\ 1 & 1 & 1 & 0\end{array}\right]+\left[\begin{array}{llll}0 & 1 & 1 & 1 \\ 1 & 1 & 1 & 0 \\ 0 & 1 & 1 & 1 \\ 1 & 1 & 1 & 0\end{array}\right]+\left[\begin{array}{llll}1 & 1 & 1 & 1 \\ 0 & 0 & 0 & 0 \\ 1 & 1 & 1 & 1 \\ 1 & 1 & 1 & 0\end{array}\right]+\circlearrowleft+\left[\begin{array}{llll}0 & 1 & 0 & 1 \\ 0 & 0 & 0 & 0 \\ 0 & 0 & 0 & 0 \\ 1 & 0 & 1 & 0\end{array}\right]+\left[\begin{array}{llll}0 & 1 & 0 & 0 \\ 0 & 1 & 0 & 0 \\ 0 & 1 & 0 & 1 \\ 1 & 0 & 1 & 0\end{array}\right]$

Multi-Layer Multileaf Collimator. Using multiple layers of leaves has been originally patented by [23] in 1997 and has been intensively studied since 2003. Most studies consider two orthogonal layers, referred in the literature as the dual-MLC $[7,12,9,8]$. Topolnjak et al. investigated the use of three layers placed every 60 degree $[18,19]$. The state-of-the-art is presented in [22] which claimed the efficiency of the gear. For example, decomposing the following intensity matrix with only $\mathrm{C} 1 \mathrm{P}$ matrices (even allowing $\mathrm{MLC}$ rotations) requires a linear number of configurations whereas only 2 are needed when using a dual-MLC. 


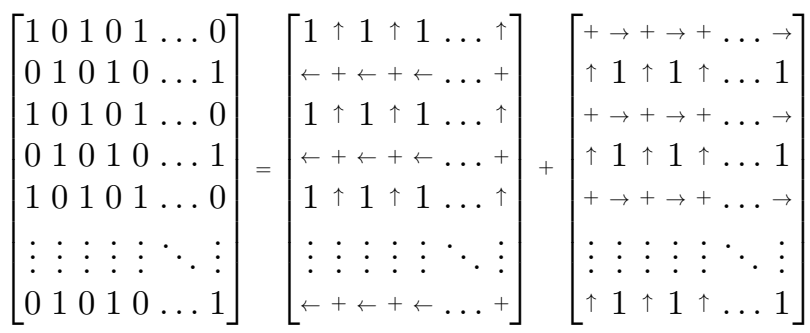

To the best of our knowledge, all these contributions mentioned the complexity increase of the problem when considering rotation or multiple layers without being able to state it formally. In this contribution, we prove formally the algorithmic hardness of the corresponding problems. More precisely, we study the Matrix Orthogonal Decomposition (MOD) problem introduced in [5] which consider the decomposition problem of an intensity matrix using a unique $90^{\circ}$ rotation and the Dual-MLC Decomposition (DMD) problem which consider two orthogonal layers of MLCs. We prove that both problems are still NP-hard when minimizing total-setup time. Finally we prove that MOD becomes NP-hard even when minimizing the total beam-on time but is approximable.

\section{Dual-MLC Decomposition minimizing total setup-time}

In order to prove the hardness of the problem, we will use the construction of Baatar et. al [1] as a gadget. Therefore, let us first present briefly a slightly modified version of their proof. As a reminder, they originally proved the hardness of total setup-time decomposition even for matrices with a single row by a reduction from the NP-complete 3 -Partition problem where one has to partition $3 Q$ numbers - say $S=\left(b_{1}, b_{2}, \ldots, b_{3 Q}\right)$ - (allowing duplicates) into $Q$ triples say $\left\{T_{1}, T_{2}, \ldots, T_{Q}\right\}$, such that each triple has the same sum. Considering that all $3 Q$ numbers sum to $N$, then every triple should have a sum of $B=\frac{N}{Q}$ (we may assume that $\frac{B}{4}<b_{i}<\frac{B}{2}$ for every $b_{i} \in S$ ).

From any instance $S$ of the 3 -Partition problem, one can construct in polynomial time an integer vector $A=x_{1} x_{2} \ldots x_{3 Q} y_{Q} y_{Q-1} \ldots y_{1} z_{0}$ such that $x_{i}=\sum_{j=1}^{i} b_{j}, y_{i}=i \cdot B$ and $z_{0}=b_{1}{ }^{1}$ and asks for a decomposition with at most $3 Q$ MLC configurations. As a reminder, any solution of the problem is a set of $\mathrm{C} 1 \mathrm{P}$ vectors (i.e. configurations) provided with corresponding intensity. Therefore, for each configuration, we will denote by left (resp. right) endpoint the first (resp. last) position of a 1 in the corresponding vector. First, notice that since $\left\{x_{i} \mid 1 \leq i \leq 3 Q\right\}$ is a set of $3 Q$ different values, any decomposition of $A$ will need at least $3 Q$ configurations and thus $3 Q$ corresponding intensities (among which one is $b_{1}$ due to $x_{1}$ ) each having their left endpoint disjointly in one of $\left\{x_{i} \mid 1 \leq i \leq 3 Q\right\}$ positions. Moreover, the configuration with the intensity $b_{1}$ is defined as a totally open configuration (i.e. a vector of $4 Q+11^{\prime} s$ ). Indeed,

\footnotetext{
${ }^{1}$ We added $z_{0}$ to the original construction for ease of proof demonstration
} 
among the at most $Q \cdot B$ radiation doses that can go through $x_{3 Q}$, exactly $B$ of them is needed for irradiating $Y_{1}$. To respect the consecutiveness of the $1^{\prime} s$, whatever left endpoints of the configurations used, the corresponding configurations will contribute exactly $B$ to each of $\left\{Y_{i} \mid 2 \leq i \leq Q\right\}$. Repeating this last argument over $\left\{Y_{i} \mid 2 \leq i \leq Q\right\}$, one can prove that each $Y_{i}$ is the right endpoints of some configurations which overall contribution sums to $B$. Moreover, by construction, $z_{0}$ needs $b_{1}$ irradiation doses that has to be included into the $B$ needed by $Y_{1}$. Consequently, since one of the configuration contributed $b_{1}$ to all positions of $A, x_{2}$ now only needs an extra $b_{2}$ contribution which should be delivered at once. Repeating this argument over $\left\{x_{i} \mid 3 \leq i \leq 3 Q\right\}$, one can prove that the set of configuration intensities is indeed $\left\{b_{i} \mid 1 \leq i \leq 3 Q\right\}$. Since, for any $1 \leq i \leq 3 Q, \frac{B}{4}<b_{i}<\frac{B}{2}$, any $Y_{j}$ will need to be the right endpoint of exactly 3 configurations to get an overall irradiation summing to $B$ (recall that there are at most $3 Q$ configurations).

Provided with these properties, one can easily prove that $(\Leftarrow)$ given a solution to the 3-Partition problem such that, w.l.o.g., $b_{1} \in T_{1}$, for all $2 \leq j \leq 3 Q$, irradiating the interval $\left[x_{j}, Y_{q}\right]$ with intensity $b_{j}$ if $b_{j} \in T_{q}$ and irradiating the full vector with $b_{1}$ leads to a valid decomposition of exactly $3 Q$ configurations. Moreover, $(\Rightarrow)$ considering any solution of the decomposition problem, defining the triples $\left\{T_{i} \mid 1 \leq i \leq Q\right\}$ such that $b_{j} \in T_{q} \Leftrightarrow$ there exists a configuration of intensity $b_{j}$ with resp. left and right endpoints in $x_{j}$ and $Y_{q}$.

Back to our original problem, we will use a slightly similar reduction using $A$ as a gadget. We, thus now consider the decomposition of a matrix. The reduction is again from the 3-Partition problem. From any instance $S$, one can construct in polynomial time a matrix $M=\left(R_{1}, R_{2}, \ldots R_{6 Q+3}\right)$ composed of $6 Q+3$ rows, where for all $1 \leq i \leq 3 Q, R_{i}=R_{6 Q+4-i}=\left(x_{3 Q+1-i}\right)^{4 Q+1}, R_{3 Q+1}=R_{3 Q+3}=$ $0^{4 Q+1}$ and $R_{3 Q+2}$ is the vector $A$ designed in the previous proof and asks again for a decomposition with at most $3 Q$ Dual-MLC configurations. Roughly, the vector $A$ is vertically surrounded by two opposed sorted heaps of vectors (increasing, when going away from $A$ ) filled with the $\left\{x_{i} \mid 1 \leq i \leq 3 Q\right\}$ values defined in the previous proof and two null rows.

$$
M=\left[\begin{array}{ccccccccc}
x_{3 Q} & x_{3 Q} & \ldots & x_{3 Q} & x_{3 Q} & x_{3 Q} & \ldots & x_{3 Q} & x_{3 Q} \\
\vdots & \vdots & \vdots & \vdots & \vdots & \vdots & \vdots & \vdots & \vdots \\
x_{2} & x_{2} & \ldots & x_{2} & x_{2} & x_{2} & \ldots & x_{2} & x_{2} \\
x_{1} & x_{1} & \ldots & x_{1} & x_{1} & x_{1} & \ldots & x_{1} & x_{1} \\
0 & 0 & \ldots & 0 & 0 & 0 & \ldots & 0 & 0 \\
x_{1} & x_{2} & \ldots & x_{3 Q} & y_{Q} & y_{Q-1} & \ldots & y_{1} & z_{0} \\
0 & 0 & \ldots & 0 & 0 & 0 & \ldots & 0 & 0 \\
x_{1} & x_{1} & \ldots & x_{1} & x_{1} & x_{1} & \ldots & x_{1} & x_{1} \\
x_{2} & x_{2} & \ldots & x_{2} & x_{2} & x_{2} & \ldots & x_{2} & x_{2} \\
\vdots & \vdots & \vdots & \vdots & \vdots & \vdots & \vdots & \vdots & \vdots \\
x_{3 Q} & x_{3 Q} & \ldots & x_{3 Q} & x_{3 Q} & x_{3 Q} & \ldots & x_{3 Q} & x_{3 Q}
\end{array}\right]
$$


The correctness of the proof relies on proving that, whereas one may use the vertical leaves to make a different set of configurations for realizing the peculiar row $A$, this would not lead to a valid solution. Indeed, the rows $R_{3 Q+1}$ and $R_{3 Q+3}$ ensures that if a vertical leaf was used to tune the irradiation configuration used for $A$ - say the one in column $j$ - then the corresponding intensity could not be used for any element of column $j$ except in $A$. Since, by construction, yet again exactly $3 Q$ configurations are required, there will exist at least one row in the end with a non null value on the column $j$. This property, ensures that if the vertical leaves are used, this is not to disturb the configurations plan of row $R_{3 Q+2}$. Provided with these properties, one can easily prove that $(\Leftarrow)$ given a solution to the 3 -Partition such that, w.l.o.g., $b_{1} \in T_{1}$, for all $2 \leq j \leq 3 Q$, irradiating the interval $R_{3 Q+2}\left[x_{j}, Y_{q}\right]$ and fully the rows $R_{3 Q-j}$ and $R_{6 Q+3-j}$ with intensity $b_{j}$ if $b_{j} \in T_{q}$ and irradiating the full rows $R_{3 Q}, R_{3 Q+2}$ and $R_{3 Q+4}$ with $b_{1}$ leads to a valid decomposition of exactly $3 Q$ configurations. Moreover, $(\Rightarrow)$ considering any solution of the decomposition problem, defining the triples $\left\{T_{i} \mid 1 \leq i \leq Q\right\}$ such that $b_{j} \in T_{q} \Leftrightarrow$ there exists a configuration of intensity $b_{j}$ with resp. left and right endpoints in $x_{j}$ and $Y_{q}$ in row $R_{3 Q+2}$ leads to a solution to 3 -Partitioning.

\section{Matrix Orthogonal Decomposition}

This section is devoted to proving a stronger result for the MOD problem: minimizing the total setup time is NP-Hard even if the intensity matrix is binary. This result shows that the problem is also NP-hard when one whants to minimize the beam-on time (whereas it is polynomial when rotation is not changed during decomposition). Fortunately, we will also prove that the problem is however approximable in this later case. For ease of presentation, we will first give a construction using an integer matrix and show afterwards how to make it binary. In order to prove the hardness of the problem, we define a reduction from the NP-Complete 3-Hitting Set problem: given a collection $C=\left\{C_{1}, \ldots, C_{m}\right\}$ of $m$ subsets of size at most three of a finite set $S=\left\{x_{1}, \ldots x_{n}\right\}$ of $n$ elements and a positive integer $k$, the problem asks for a subset $S^{\prime} \subseteq S$ with $\left|S^{\prime}\right| \leq k$ such that $S^{\prime}$ contains at least one element from each $C_{i}$ 's.

From any instance $(C, k)$ of the 3 -Hitting Set problem, one can construct in polynomial time a square matrix $M$ composed of two rows and columns independant submatrices - a submatrix of $2 n+9$ columns and $2 n+8$ rows referred as $M_{D H V}$ defined below is placed top-right of $M$ whereas another submatrix of $3 n+2$ columns and $m+4$ rows named $M_{3 H S}$ and defined later on is placed bottom-left; the rest of the matrix $M$ is filled with 0's in order to obtain a square matrix - and asks for a decomposition with at most $n+3$ MLC configurations.

The submatrix $M_{D V H}$ is designed in such a way that it will ensure that any solution to the decomposition problem will use only one vertical configuration and $(n+2)$ horizontal ones. Indeed, since, by construction, a) there are $(n+4)$ horizontal blocks of single $1^{\prime} s$ in the first row, and b) all the $2(n+2)$ last columns are each composed of $(n+4)$ vertical blocks of single $1^{\prime} s$, any solution (i.e. not 
inducing more than $(n+3)$ configurations) has to have at least one horizontal and one vertical configurations. Moreover, any solution has to use exactly one vertical configuration. Indeed, suppose, aiming at a contradiction, that a given solution uses more - say $k^{\prime}$ vertical configurations, then there at most $k^{\prime} 1^{\prime} s$ from each column that can be irradiated by those $k^{\prime}$ configurations. Unfortunately, since $k^{\prime} \leq(n+2)$, at least two $1^{\prime} s$ per column (except the five leftmost ones) will subsist. In order for the solution to be feasible, one would then have to irradiate the remaining $1^{\prime} s$ with a unique horizontal configuration. To do so, the remaining $1^{\prime} s$ should be placed in order not to have more than one 1 per row; a contradiction since we have at least $2 \times(2 n+4) 1^{\prime} s$ and at most $2 n+8$ rows. We just proved that the submatrix $M_{D V H}$ will force any solution to use exactly one vertical configuration and $(n+2)$ horizontal ones, each with an intensity of 1.

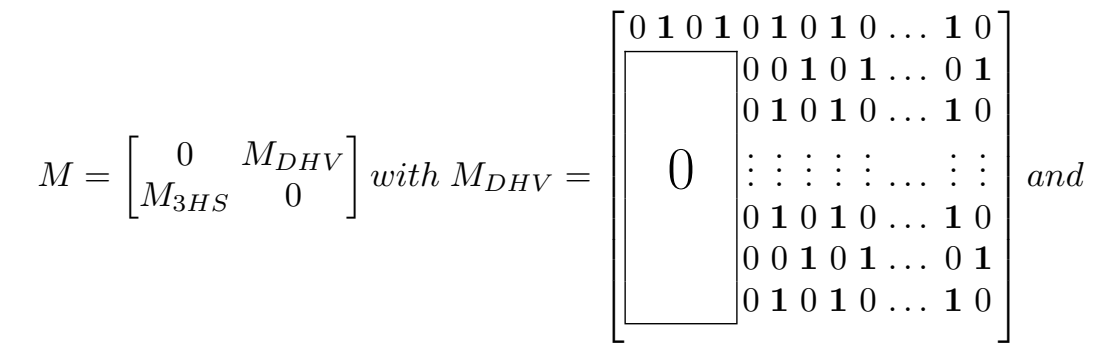

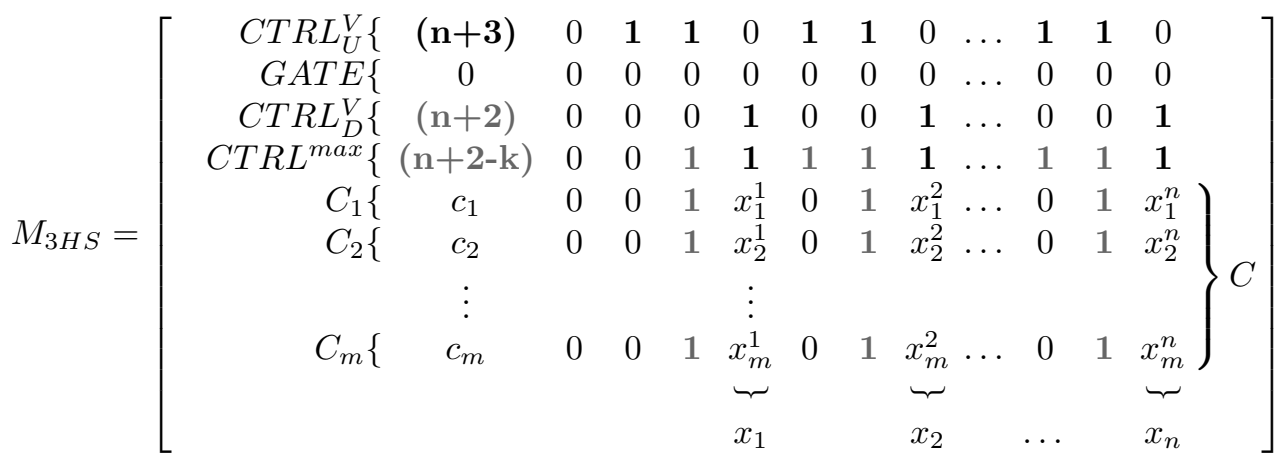

Let us now describe the submatrix $M_{3 H S}$ which is totally independant of $M_{D V H}$ but which will inherit the repartition of the vertical and horizontal configurations that we just showed. $M_{3 H S}$ is defined as pictured above and where $x_{i}^{j}=\mathbf{2}$ if $x_{j} \in C_{i} ; x_{i}^{j}=\mathbf{1}$ otherwise and $c_{i}=0$ if $\left|C_{i}\right|=3 ; c_{i}=1$ otherwise. The submatrix $M_{3 H S}$ is designed to encode the 3-Hitting Set instance. Roughly, each subset $C_{i}$ of $C$ is encoded by a row whereas each element $x_{i}$ of $S$ is encoded by a column ${ }^{2}$. Let us now prove some interesting properties of this construction.

Let us have a look at the constraints of the unique vertical configuration. Since $C T R L_{U}^{V}[0]$ is set to $(n+3)$ - that is both the maximal number of configu-

\footnotetext{
${ }^{2}$ Due to space consideration, we will not include a full illustration of a construction but one may build one from our website http://igm.univ-mlv.fr/ gblin/MOD/index.php.
} 
rations and intensity, the first column of the vertical configuration will have to irradiate this last and so does the corresponding row $\left(i . e .(2 n+9)^{\text {th }}\right.$ of $\left.M\right)$ of all the horizontal configurations. It has many consequences: a) all the $1^{\prime} s$ of $C T R L_{U}^{V}$ will have to be irradiated during the vertical configuration (inducing that no other $1^{\prime} s$ in the corresponding columns can be irradiated during the vertical configuration - namely columns in $\{3 j-1,3 j \mid 1 \leq j \leq n\})$ and b) $C T R L_{D}^{V}[0], C T R L^{\max }[0]$ and any $C_{i}[0]$ will all only be irradiated by horizontal configurations ${ }^{3}$. Since $C T R L_{D}^{V}[0]$ is set to $(n+2)$, all the horizontal configurations for this specific row $C T R L_{D}^{V}$ will need to be dedicated to $C T R L_{D}^{V}[0]$. This implies, in turn, that all remaining $1^{\prime} s$ of $C T R L_{D}^{V}$ - that is $\left\{C T R L_{D}^{V}[i] \mid i=2+3 j, 1 \leq j \leq n\right\}$ - would have to be vertically irradiated. On the whole, except for the set of bottom leaves for the columns $\{i=2+3 j \mid 1 \leq j \leq n\}$, we know exactly what is the endpoint positions of each leave (top and bottom) of the vertical configuration in $M_{3 H S}$ : a) for all $i \in\{0,3 j, 3 j-1 \mid 1 \leq j \leq n\}$ the $i^{\text {th }}$ top leave (resp. bottom one) precisely blocks all the rows preceding (resp. succeeding) $C T R L_{U}^{V}$, b) column 1 is totally blocked and c) for all $i \in\{3 j+1 \mid 1 \leq j \leq n\}$ the $i^{\text {th }}$ top leave precisely blocks all the rows preceding $C T R L_{D}^{V}$.

Now, notice that any $C_{i}$ needs at least $(n+3)$ configurations to be realized. This implies that any $C_{i}$ should be irradiated at least in one of its column by the vertical configuration. By construction, this irradiation can only occur in $\left(x_{i}^{j}\right)^{\prime} s$ positions (i.e. $\{3 j+1 \mid 1 \leq j \leq n\}$ ) moreover set to $\mathbf{2}$ (otherwise it will not help decreasing the total irradiation needed to realize $C_{i}$ ) - later referred to as target positions. The $C T R L^{\max }$ row is designed to ensure that at least $n-k$ cells among $\left\{C T R L^{\max }[3 j+1] \mid 1 \leq j \leq n\right\}$ will be blocked by bottom leaves. In other words, at least $n-k$ bottom leaves will block all the succeeding rows of $C T R L^{\max }$. Thus, at most $k$ bottom leaves would be able to allow vertical irradiation contribution for the target positions. As we just prove, the only differences between both solutions to the decomposition problem is the position of bottom leaves endpoints for positions in $\{3 j+1 \mid 1 \leq j \leq n\}$; we will thus characterize any such solution as a set of $n$ positions in $[2 n+13,2 n+m+14]$ (corresponding to all possible solutions $-2 n+m+14$ being a leave not used at all).

Provided with these properties, one can easily prove that $(\Leftarrow)$ given a solution $\left(S^{\prime} \subseteq S\right)$ to 3 -Hitting Set problem, for each $1 \leq i \leq n$, if $x_{i} \in S^{\prime}, P[i]=$ $2 n+m+14 ; P[i]=2 n+13$ otherwise. We claim that $P$ corresponds to valid positions for the bottom leaves in $\{3 j+1 \mid 1 \leq j \leq n\}$. Since $S^{\prime}$ is a hitting set of size at most $k$, we can ensure that at least one element of each subset in $C$ belongs to $S^{\prime}$. This guarantees that all $C_{i}^{\prime} s$ rows and $C T R L^{\max }$ are realized. Moreover, $(\Rightarrow)$ considering any solution to the decomposition problem, one can define the hitting set $S^{\prime}$ such that $x_{j} \in S^{\prime} \Leftrightarrow$ the position of the $(3 j+1)^{\text {th }}$ bottom leaf is strictly greater than $2 n+13$.

Let us now try to transform the construction in order to obtain a binary matrix with the same property. First, on can encode the $x_{i}^{\prime} s$ using two columns rather than one as follows. Insert a column just before each actual column rep-

\footnotetext{
${ }^{3}$ For ease, in the above description of $M_{3 H S}$ matrix, all the cells which will not have a contribution from the vertical configuration have been put in gray.
} 
resenting an $x_{i}$, fill it with $0^{\prime} s$ except on the $C T R L_{U}^{V}$ row which has to be set to 1 and the $C_{j}^{\prime} s$ rows where the corresponding columns have to be set to $0 \mathbf{1}$ if $x_{i} \in C_{j} ; 11$ otherwise ${ }^{4}$. This update is clearly not changing the original proof. The tricky part stands in the replacement of $C T R L_{U}^{V}[0]$. Indeed, one wants that it still requires all the horizontal configurations and the vertical one. To do so, one can design a submatrix of $2(n+4)$ rows defined as follows: a) each odd row is filled with $0^{\prime} s$ and $\mathrm{b}$ ) the $i^{\text {th }}$ even row is defined as $(0)^{i-1} \mathbf{1} 0(10)^{n+2}(0)^{n+3-i}$. Roughly, the block representing $(n+3)\left(\right.$ i.e. $\left.\left(\begin{array}{ll}1 & 0\end{array}\right)^{n+3}\right)$ is shifted right of one position every new even row. This ensures that no one under the position of this gadget will be able to have a vertical contribution and that in any of the corresponding rows, all the remaining $1^{\prime} s$ will need to be irradiated vertically. We just showed that the properties of the original gadget are preserved. Both $C T R L_{D}^{V}[0]$ and $C T R L^{\max }[0]$ can be easily replaced by resp. $(01)^{n+2}(0)^{3 n+7}$ and $\left(\begin{array}{ll}0 & 1\end{array}\right)^{n+2-k}(0)^{3 n+7}$. Again, the properties of the original gadgets are preserved. This concludes the proof of the following theorem.

Theorem 1. The Matrix Orthogonal Decomposition problem is NP-hard when minimizing either the total setup or the total beam-on time.

Now, let us prove that there exists an interesting algorithm based on linear programming and rounding techniques that produces an approximate solution for minimizing the total beam-on time. First, recall that for horizontal configurations, rows can be dealt with separately. It is also the case for vertical configurations and columns. Indeed, an intensity matrix is realized by a sequence of MLC configurations each of which is maintained for a certain amount of time (corresponding to the intensity). Since the problem is to minimize the sum of intensities and not the number of configurations, one can always consider that configurations can be changed every unit of time. This implies that any row (column) can be processed independently of the others and that the overall beam-on time will be deduced by the (most) expensive row (column).

The problem can be phrased, as an Integer Linear Programming, as follows:

$$
\begin{array}{lll}
\operatorname{minimize} & H+V & \\
\text { subject to } & \forall 1 \leq k \leq m, \quad \sum_{i \leq j} H_{i j}^{k} \leq H(1) \\
& \forall 1 \leq k \leq m, \quad \sum_{i \leq j}^{k} V_{i j}^{k} \leq V(2) \\
& \forall k, k^{\prime} \in\{1, \ldots m\}^{2}, \sum_{i \leq k^{\prime} \leq j} H_{i j}^{k}+\sum_{i^{\prime} \leq k \leq j^{\prime}} V_{i^{\prime} j^{\prime}}^{k^{\prime}}=M[k]\left[k^{\prime}\right](3) \\
& \forall i, j, k, \quad H_{i j}^{k} \geq 0, V_{i j}^{k} \geq 0 \\
& 1 \leq i \leq j \leq m, 1 \leq i^{\prime} \leq j^{\prime} \leq m, H \geq 0, V \geq 0 .
\end{array}
$$

For any row of the intensity matrix $M$, let $H_{i j}^{k}$ be a variable indicating the amount of time the following horizontal configuration is maintained: considering the $k^{\text {th }}$ pair of leaves, the left one's endpoint is at position $i-1$ and the right one's

\footnotetext{
${ }^{4}$ Due to space consideration, please consider checking the construction on our website http://igm. univ-mlv.fr/ gblin/MOD/index.php.
} 
endpoint is at position $j+1$ (therefore irradiating any position between $i$ and $j$ in row $k$ ). Similarly, for any column of the intensity matrix $M$, let $V_{i j}^{k}$ be a variable indicating the amount of time the following vertical configuration is maintained: considering the $k^{\text {th }}$ pair of leaves, the left one's endpoint is at position $i-1$ and the right one's endpoint is at position $j+1$ (therefore irradiating any position between $i$ and $j$ in column $k$ ). Finally, variables $H$ and $V$ are respectively horizontal and vertical costs of a solution computed respectively as $\max _{k} \sum_{i \leq j} H_{i j}^{k}$ and $\max _{k} \sum_{i \leq j} V_{i j}^{k}$ (which is encoded by constraints (1) and (2)). Constraint (3) ensures that the desired intensity matrix is realized. Indeed, $\sum_{i \leq k^{\prime} \leq j} H_{i j}^{k}$ (resp. $\left.\sum_{i^{\prime} \leq k \leq j^{\prime}} V_{i^{\prime} j^{\prime}}^{k^{\prime}}\right)$ represents the overall contribution of all the horizontal (resp. vertical) configurations contributing to the entry $M[k]\left[k^{\prime}\right]$. There are about $2 m^{3}+2$ variables, $2 n$ inequalities and $n^{2}$ equalities. Our linear programming problem can be rewritten with only inequalities. Indeed, each equality constraint may be removed, by solving it for variable $H_{0 k^{\prime}}^{k}$ and substituting this solution into the corresponding form of constraint (1) (i.e. for the corresponding $k$ ).

Of course Integer Linear Programming is NP-hard. Therefore, we relax the integrality constraint, that is, allowing all variables to take a non-integral but still positive value. We end-up with a fractional linear program that can be solved in polynomial time. Notice that the solution provided by this linear program cannot be greater than the optimal integer one, since we only allow more solutions to become feasible. We apply a clever rounding of the fractional solution to obtain an integral feasible solution not too far from optimal.

Assume that $f_{L}:\left\{V, H, H_{i j}^{k}, V_{i j}^{k} \mid 1 \leq i \leq j \leq m, 1 \leq k \leq m\right\} \rightarrow \mathbb{R}$ is an optimal fractional solution of the relaxed version of our problem. If one slightly modifies the values of $H_{i j}^{k}{ }^{\prime} s$ then due to constraint (3) the values of $V_{i^{\prime} j^{\prime}}^{k^{\prime}}{ }^{\prime} s$ will need to be modified accordingly and with a comparable amount. The basic idea is to provide an integral rounding of the horizontal configurations and compute polynomially the corresponding vertical configurations while guaranteeing that the corresponding solution is a good approximation of the optimal one.

Let us present the rounding technique for a single row - say the $k^{\text {th }}$. Considering all the corresponding variables $\left\{H_{i j}^{k} \mid 1 \leq i \leq j \leq m\right\}$, one can represent each non-null variable $H_{i j}^{k}$ by an interval $[i, j]$ over the real line on $[1, m]$ weighted by $H_{i j}^{k}$ (illustrated in Figure $2^{5}$ ). Let us transform this set of intervals $\mathcal{I}$ into a set $\mathcal{I}^{\prime}$ where given any pair of intervals either one is included into the other or they are disjoint. To do so, we process $\mathcal{I}$ with the following algorithm. While there exists two intervals $[i, j]$ and $[k, l]$ with respective weights $w_{1}$ and $w_{2}$ such that $i<k<j<l$ (i.e. crossing) remove $[i, j]$ and $[k, l]$ from $\mathcal{I}$ and add $[i, k-1]$, $[k, j]$ and $[j+1, l]$ with respective weights $w_{1}, w_{1}+w_{2}$ and $w_{2}$. As an example, given intervals $[1,9]$ and $[3,11]$, it will result in intervals $[1,2],[3,9]$ and $[10,11]$ with respectively weights $H_{1,9}^{k}, H_{1,9}^{k}+H_{3,11}^{k}$ and $H_{3,11}^{k}$. Now that all intervals are nested or independent, while there exists three intervals $[x, y],[i, j]$ and $[k, l]$ with respective weights $w_{1}, w_{2}$ and $w_{3}$ such that $x \leq i<j \leq k<l \leq y$, if $j<k$ then remove $[x, y]$ from $\mathcal{I}$ and add $[x, j]$ and $[j, y]$ both weighted by $w_{1}$;

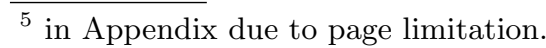


otherwise $(j=k)$ remove $[i, j]$ and $[k, l]$ from $\mathcal{I}$ and if $w_{2}<w 3$ then add $[i, l]$ and $[k, l]$ with respective weights $w_{2}$ and $w_{3}-w_{2}$; otherwise add $[i, l]$ and $[i, j]$ with respective weights $w_{3}$ and $w_{2}-w_{3}$. As an example, given intervals [3,9], $[4,7]$ and $[9,9]$, it will result in intervals $[3,7],[8,9],[4,7]$ and $[9,9]$. Moreover given two copies of an interval $[i, j]$ with respective weights $w_{1}$ and $w_{2}$, remove them and add an interval $[i, j]$ with weight $w_{1}+w_{2}$. As an example, copies of intervals $[1,2],[3,7]$ and $[8,9]$ have been merged.

We end up with a set of independent subset of nested intervals ( later referred to as a stack). Note that there are at most $m$ such stacks. We will proceed to the rounding of each stack separately. We will do so while ensuring that the sum of the original weights is smaller than the sum of the rounded ones with a gap of at most 1 . This will induce that for a given row of the horizontal configuration, we manage to get an integral solution with an at most $m$ extra cost. For ease, considering the stack as increasingly sorted by interval size and let $w_{i}$ and $w_{i}^{\prime}$ denote respectively the original and rounded weights of the $i^{\text {th }}$ interval of the stack. The rounding algorithm proceeds as follows: start from the wider interval and round up $w_{1}{ }^{6}$. Then consider iteratively each remaining interval - say the $j^{\text {th }}$, round it down if possible - that is if $\left\lfloor w_{j}\right\rfloor+\sum_{i=1}^{j-1} w_{i}^{\prime} \geq \sum_{i=1}^{j} w_{i}$-round it up otherwise. As an example, consider

\begin{tabular}{|c|c|c|c|c|c|c|}
\hline$j$ & 1 & 2 & 3 & 4 & 5 & 6 \\
\hline$w_{j}$ & 3.3 & 1.2 & 2.4 & 1.5 & 4.5 & 2.5 \\
\hline$\sum_{i=1}^{j} w_{i}$ & 3.3 & 4.5 & 6.9 & 8.4 & 12.9 & 15.4 \\
\hline$w_{j}^{\prime}$ & 4 & 1 & 2 & 2 & 4 & 3 \\
\hline$\sum_{i=1}^{j} w_{i}^{\prime}$ & 4 & 5 & 7 & 9 & 13 & 16 \\
\hline
\end{tabular}

Applying the rounding to each row of the horizontal configurations leads to an integral solution for the horizontal configurations that we can subtract for the original intensity matrix.Then, we compute in polynomial time the vertical configurations on the resulting matrix. We claim that the overall solution is at most $2 m$ from the optimal solution. Indeed, in the resulting matrix, each cell is at most greater by one than the fractional matrix. This means that the sum of the elements in any column is at most greater by $m$ than the fractional matrix. Thus we loose at most $m$ with the rounding of the horizontal configurations plus at most $m$ for adjusting the vertical configurations, for a total of an additive $2 m$ overcost.

\section{References}

1. D Baatar, H.W Hamacher, M Ehrgott, and G.J Woeginger. Decomposition of integer matrices and multileaf collimator sequencing. DAM, 152(1-3):6-34, 2005.

2. A.W Beavis, P.S Ganney, V.J Whitton, and L Xing. Optimisation of MLC orientation to improve accuracy in the static field delivery of IMRT. Proc. of 22nd IEEE Engineering in Medicine and Biology Society, 4:3086-3089, 2000.

${ }^{6}$ One cannot do otherwise since there exists at least one position not covered by another interval. 
3. M Broderick, M Leech, and M Coffey. Direct aperture optimization as a means of reducing the complexity of Intensity Modulated Radiation Therapy plans. Radiation oncology, 4:8, 2009.

4. R Burkard. Open problem session, Oberwolfach Conference on Combinatorial Optimization.

5. X Dou, X Wu, JE Bayouth, and JM Buatti. The Matrix Orthogonal Decomposition Problem in Intensity-Modulated Radiation Therapy. Computing and Combinatorics, pages 156-165, 2006.

6. H.W Hamacher and K-H Küfer. Inverse radiation therapy planning - a multiple objective optimization approach. DAM, 118(1-2):145-161, 2002.

7. JH Hughes. US Patent 6,600,810: Multiple layer multileaf collimator design to improve resolution and reduce leakage, 2003.

8. Y Liu, C Shi, B Lin, and CS Ha. Delivery of four-dimensional radiotherapy with TrackBeam for moving target using a dual-layer MLC: dynamic phantoms study. Journ. of Applied Clinical Medical Physics, 10(2):1-21, 2009.

9. Y Liu, C Shi, P Tynan, and N Papanikolaou. Dosimetric characteristics of duallayer multileaf collimation for small-field and intensity-modulated radiation therapy applications. Journ. of Applied Clinical Medical Physics, 9(2):2709, 2008.

10. M Milette, M Rolles, and K Otto. TU-C-224A-06: Exploiting the Full Potential of MLC Based Aperture Optimization Through Collimator Rotation. Medical Physics, 33(6):2191, 2006.

11. MP Milette. Direct optimization of $3 D$ dose distributions using collimator rotation. $\mathrm{PhD}$ thesis, 2008.

12. S Oh, W Jung, and T Suh. SU-FF-T-28: A New Concept of Multileaf Collimator (dual-Layer MLC). Medical Physics, 34(6):2407, 2007.

13. K Otto. Intensity modulation of therapeutic photon beams using a rotating multileaf collimator. PhD thesis, University Of British Columbia, 2003.

14. K Otto. US Patent 6,907,105: Methods and apparatus for planning and delivering intensity modulated radiation fields with a rotating multileaf collimator, 2005.

15. K Otto and BG Clark. Enhancement of IMRT delivery through MLC rotation. Physics in medicine and biology, 47(22):3997-4017, 2002.

16. K Otto, M Milette, and M Schmuland. SU-FF-T-104: Rotating Aperture Optimization - Planning and Delivery Characteristics. Medical Physics, 32(6):1973, 2005.

17. ML Schmuland. Dose verification of rotating collimator intensity modulated radiation thearpy. PhD thesis, 2006.

18. R Topolnjak, U a Van Der Heide, B W Raaymakers, a N T J Kotte, J Welleweerd, and J J W Lagendijk. A six-bank multi-leaf system for high precision shaping of large fields. Phys. in Medicine and Biology, 49(12):2645-2656, 2004.

19. R Topolnjak, U a van der Heide, and J J W Lagendijk. IMRT sequencing for a six-bank multi-leaf system. Physics in medicine and biology, 50(9):2015-31, 2005.

20. DQ Wang, RW Hill, and SC Lam. US Patent 7,015,490: Method and apparatus for optimization of collimator angles in intensity modulated radiation therapy treatment, 2006.

21. S Webb. Does the option to rotate the Elekta Beam Modulator MLC during VMAT IMRT delivery confer advantage? - a study of 'parked gaps'. Physics in medicine and biology, 55(11):N303-19, 2010.

22. S Webb. A 4-bank multileaf collimator provides a decomposition advantage for delivering intensity-modulated beams by step-and-shoot. Physica medica, 28(1):16, 2012.

23. JY Yao. US Patent 5,591,983: Multiple layer multileaf collimator, 1997. 


\section{Appendix}

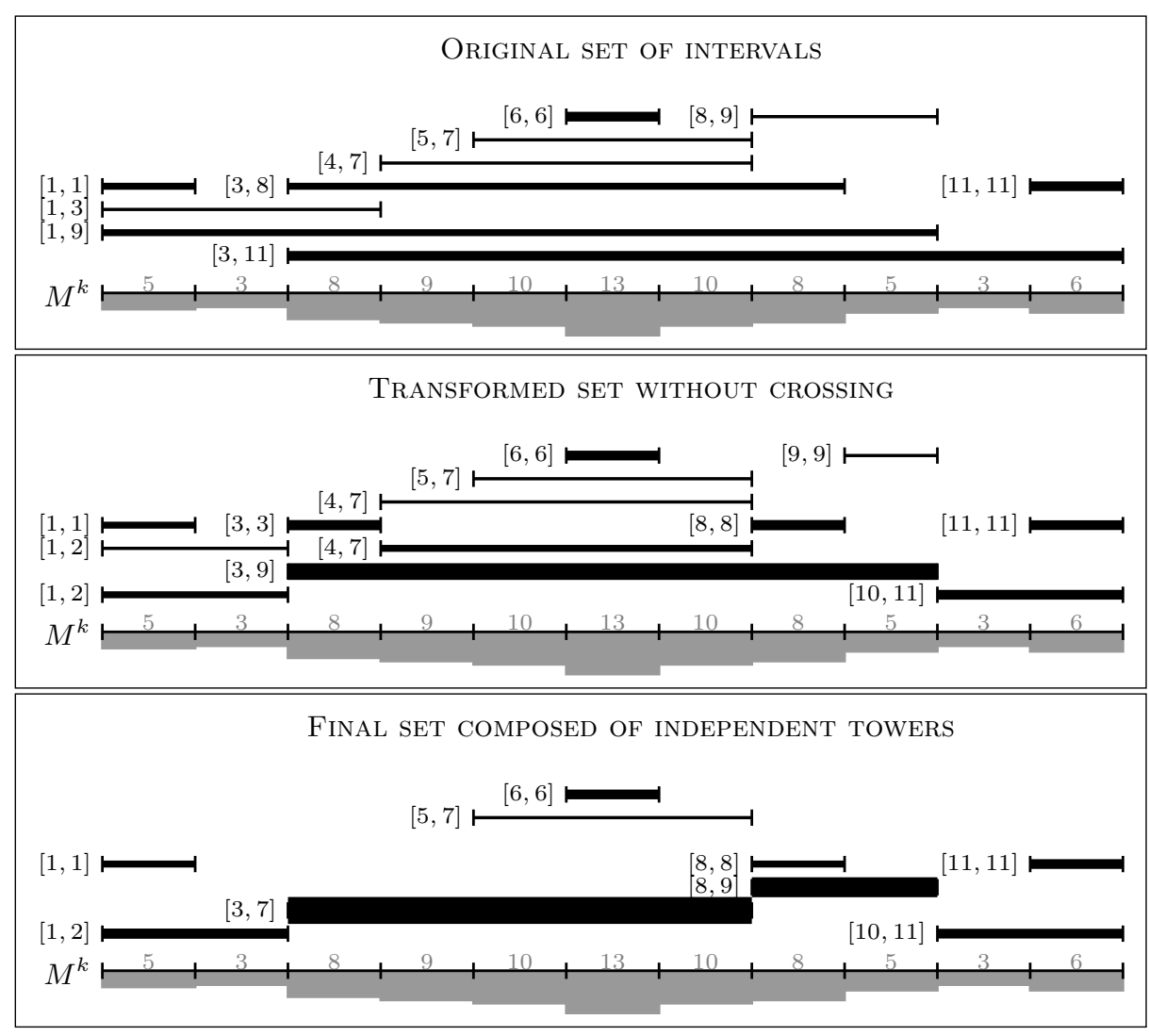

Fig. 2. 